\title{
Doping Induced Abnormal Contraction and Significant Reduction of Lattice Thermal Conductivity of Open Framework $\mathrm{Si}_{24}$
}

\author{
Tao Ouyang ${ }^{1 *}$, Qingyi Liu ${ }^{1}$, Mingxing Chen ${ }^{2}$, Chao Tang ${ }^{1}$, Jin $\mathrm{Li}^{1}$, Chunxiao Zhang ${ }^{1}$, Chaoyu He${ }^{1}$, Hua Bao ${ }^{3^{*}}$, Jianxin Zhong ${ }^{1}$ and Ming $\mathrm{Hu}^{4 *}$
}

Effectively suppressing lattice thermal conductivity is a critical step for improving the thermoelectric performance. Taking open framework $\mathrm{Si}_{24}$, a potential thermoelectric material and a newly synthesized cage-like Si allotrope, as an example, we systematically investigate the lattice thermal conductivity of this new structure filled with alkaline-earth guest atoms (X@Si $\mathrm{Si}_{24}, \mathrm{X}=\mathrm{Mg}, \mathrm{Ca}, \mathrm{Sr}$, and $\mathrm{Ba}$ ) by combining first-principles calculation and phonon Boltzmann transport theory. The calculated lattice thermal conductivity is obviously decreased as guest atoms are inserted in the void sites of the $\mathrm{Si}_{24}$ framework, which is a common phenomenon for the guest-host systems. However, it is surprising to find that the thermal conductivity of this new filler structure presents a prominent element dependency. Inserting $\mathrm{Ca}$ into $\mathrm{Si}_{24}$ framework generally leads to 3 to 10 times lower thermal conductivity than that with other alkaline-earth atom fillers, and the value along $z z$ crystal direction of $0.59 \mathrm{~W} / \mathrm{mK}$ is even lower than that of amorphous silicon, despite the intrinsic thermal conductivity of pristine $\mathrm{Si}_{24}$ is as high as $21.25 \mathrm{~W} / \mathrm{mK}$. Such ultra-low thermal conductivity is found to be closely related to the strong harmonic interatomic interaction among guest and host atoms of Ca@Si $\mathrm{Si}_{24}$ system. The strong interaction gives rise to anomalous contraction effect on the $\mathrm{Si}_{24}$ lattice (the volume abnormally decreases) and more dispersive phonon branches in low frequency range, which boosts the three-phonon scattering channels (reflected by the weighted phase space) and eventually suppresses the thermal conductivity of $\mathrm{Si}_{24}$. Finally, based on a simple and effective lattice chain model, we reproduce the abnormal thermal phenomenon observed in $\mathrm{Ca} @ \mathrm{Si}_{24}$, and further demonstrate that the origin stems from the strong interaction between Ca and $\mathrm{Si}$ atoms. These findings shed light on a new physical mechanism for the reduction in thermal conductivity of $\mathrm{Ca} @ \mathrm{Si}_{24}$, which offer a promising approach to improve the thermoelectric efficiency of $\mathrm{Si}_{24}$ related materials.

Keywords: Open framework Si; Lattice thermal conductivity; Doping; Thermoelectrics

Received 8 November 2018, Accepted 15 December 2018

DOI: $10.30919 /$ esee8c210

\section{Introduction}

Owing to the increasingly severe energy and environmental crisis, exploration of new renewable and clean energy has aroused considerable attentions recently. ${ }^{1-3}$ Thermoelectricity, which could potentially convert part of low-grade waste heat into viable electricity, is one of the pollution free energy conversion approaches. ${ }^{3-8}$ The performance of thermoelectric materials is measured via the dimensionless figure of merit: $Z T=S^{2} \sigma T / \kappa$. Here the numerator contains Seebeck coefficient $S$, electronic conductivity $\sigma$, and given temperature $T$, while denominator $\kappa$ is the thermal conductivity composed of the contributions from phonons $\kappa_{p}$ and electrons $\kappa_{e}$. Obviously, to achieve

${ }^{1}$ Hunan Key Laboratory for Micro-Nano Energy Materials and Device and School of Physics and Optoelectronics, Xiangtan University, Xiangtan 411105, Hunan, China

${ }^{2}$ College of Physics and Information Science, Hunan Normal University, Changsha 410081, China

${ }^{3}$ University of Michigan-Shanghai Jiao Tong University Joint Institute, Shanghai Jiao Tong University, Shanghai 200240, China

${ }^{4}$ Department of Mechanical Engineering, University of South Carolina, Columbia, SC 29208, USA

*E-mail: ouyangtao@xtu.edu.cn; hua.bao@sjtu.edu.cn; hu@sc.edu high thermoelectric performance, a material should possess excellent electronic transport and inefficient thermal transport ability. However, it is a great challenge to satisfy such conflicting condition in conventional bulk systems because the transport parameters listed above are strongly coupled with each other. ${ }^{9}$ Therefore, searching and optimizing efficient thermoelectric materials becomes a hot topic in present thermoelectric community.

Silicon ( $\mathrm{Si}$ ) is the $14^{\text {th }}$ element on the periodic table and the second most abundant element in the Earth's crust. Due to the economic applicability and mature preparation technology, diamond cubic silicon (DC-Si) is the mainstay and building block of current semiconductor and photovoltaic industries. However, such relevant material is not a suitable candidate for thermoelectric applications (the $Z T$ is merely 0.01 at room temperature), ${ }^{10-12}$ which is mainly originated from the broad spectral distribution of phonons and high lattice thermal conductivity $(140 \mathrm{~W} / \mathrm{mK}) .^{13}$ Actually, in addition to DC-Si, several new and metastable silicon crystal structures at ambient conditions have also been predicted and synthesized in experiment, ${ }^{124}$ e.g., rhombohedral structured Si (R8-Si), ${ }^{19}$ body-centered structured Si (BC8-Si), ${ }^{20}$ bodycentered tetragonal structured $\mathrm{Si}$ (Bct-Si), ${ }^{21}$ and various Si-based clathrates. ${ }^{22}$ Among these silicon allotropes, the Si-based clathrates are promising candidates for thermoelectrics. This is because such open framework structures could provide enough void space for guest atoms or molecules and build so called host-guest systems, which fulfills the requirement of 'phonon-glass and electron-crystal' thermoelectric model 
proposed by Slack. ${ }^{25}$

Recently, a new open framework silicon allotrope has been successfully produced through using a high-pressure precursor method. ${ }^{26}$ This system, named $\mathrm{Si}_{24}$, contains 24 silicon atoms occupying three symmetric Wyckoff positions $(0,0.24285,0.55476),(0,0.57130$, $0.34274)$, and $(0,0.02862,0.59056)$ in an orthorhombic (space group $\mathrm{Cmcm}$, No. 63) primitive unit cell. Such new silicon allotrope not only possesses unique crystal structure, but also shares eximious electronic and thermodynamic properties. ${ }^{26}$ For example, $\mathrm{Si}_{24}$ is quasi-direct bandgap semiconductor (bandgap is about $1.3 \mathrm{eV}$ ) which is suitable for solar harvesting. Meanwhile, the $\mathrm{Si}_{24}$ is predicted to be stable in a wide range of temperature (about $750 \mathrm{~K}$ ) and pressure (about 8.0 GPa). The cage-liked structure and fascinating physical properties imply that $\mathrm{Si}_{24}$ might have prominent thermoelectric performance and could be a competitive candidate for fabrication of Si-based thermoelectric devices. Recent theoretical studies further demonstrate this conjecture. ${ }^{27}{ }^{28}$ By using first-principles calculations, people reveal that the thermoelectric figure of merit of $\mathrm{Si}_{24}$ (approach 0.3 at room temperature) is almost an order of magnitude higher than that of DC-Si. ${ }^{2728}$ However, the thermoelectric conversion efficiency of $\mathrm{Si}_{24}$ still cannot satisfy the requirement of practical applications ( $Z T$ uld larger than 1.0), and the major obstacle is high lattice thermal conductivity (about $45 \mathrm{~W} / \mathrm{mK}$ at room temperature). In order to further improve the thermoelectric performance of $\mathrm{Si}_{24}$, effectively reducing its lattice thermal conductivity is of crucial importance.

It is well known that inserting guest atom in open framework structure is one of the key approaches to reduce the thermal conductivity, while the underlying mechanism is still contentious up to now. ${ }^{29.38}$ Some studies suggest that the reduction of phonon group velocity, attributed to the mode hybridization between the local vibrational of guest atoms (rattler modes) and acoustic heat carrying phonons propagating through the host framework, is responsible for the low lattice thermal conductivity. ${ }^{29.32}$ In contrast to the rattling concept, other inelastic neutron scattering (INS) experimental and theoretical works point out that the principal effect of the guest atoms is to enhance the phonon anharmonic effects and suppress the phonon relaxation time, ${ }^{33.35}$ which gives rise to the decrease of lattice thermal conductivity. These works with different conclusions indicate that more and deeper explorations are indispensable to clarify the role of guest atoms on the diminution of lattice thermal conductivity of open framework systems.

For the purpose of enhancing the thermoelectric performance of $\mathrm{Si}_{24}$ and clarifying the underlying mechanism, in this paper, we present a systematic investigation of a first-principle transport calculation on lattice thermal conductivity of $\mathrm{Si}_{24}$ filled with the group of alkaline-earth atoms. The results show that the thermal transport of pristine $\mathrm{Si}_{24}$ could be obviously suppressed upon filling guest atoms and present prominent element dependency. Among the four types of alkaline-earth atom fillers, the thermal conductivity could be dramatically reduced as $\mathrm{Ca}$ atoms are inserted to the void site of $\mathrm{Si}_{24}$, which is several ten times smaller than of pristine $\mathrm{Si}_{24}$ and even lower than that of amorphous silicon (along $z z$ crystal direction). Such ultra-low thermal conductivity mainly originates from the strong harmonic atomic interaction among guest and host atoms of $\mathrm{Ca} @ \mathrm{Si}_{24}$ structure. This paper is organized as follows. In Section II, a brief description about the calculation method and detail employed in this work is given. In Section III, we present the lattice thermal conductivity results and discussion of these related to microscopic phonon mode properties and electron distribution. A simplified lattice chain model is developed to further elucidate the origin of the abnormal low lattice thermal conductivity observed in $\mathrm{Ca} @ \mathrm{Si}_{24}$. Finally, our concluding remarks of this work are summarized in Section IV.

\section{Model and method}

In the present work, the first-principles calculations are performed within the framework of density functional theory (DFT), as implemented in the Vienna $a b$ initio simulation package (VASP). ${ }^{39}$ The Projector augmented wave (PAW) pseudo-potentials ${ }^{40,41}$ are used for the interaction among atoms and Ceperley-Alder local-density approximation $(\mathrm{LDA})^{42}$ is adopted for exchange and correlation function. The kinetic energy cut-off of the plane-wave basis is set at 400 $\mathrm{eV}$ and the Brillouin zones of primitive cell are sampled with Monkhorst-Pack k-meshes of $21 \times 7 \times 7$. The internal coordinates and lattice constants are fully optimized through the conjugate gradient algorithm until the maximum Hellman-Feynman force on each atom is smaller than $1 \times 10^{-5} \mathrm{eV} / \AA$.

The phonon transport properties are evaluated by means of phonon Boltzmann transport equation (PBTE) as implemented in the ShengBTE package ${ }^{43}$ with harmonic second-order interatomic force constants $\left(2^{\text {nd }}\right.$ IFCs) and anharmonic third-order IFCs ( $3^{\text {rd }}$ IFCs) as inputs. The phonon dispersions and $2^{\text {nd }}$ IFCs of the calculated systems are calculated by using VASP and PHONONPY packages ${ }^{44}$ with $3 \times 1 \times 1$ supercell. The corresponding numbers of k-mesh are accordingly scaled down compared with the case of primitive cell calculation. The anharmonic $3^{\text {rd }}$ IFCs, including the information of phonon-phonon scatterings, are calculated using same supercell with cutoff of interaction range up to sixth nearest neighbors. Taking the symmetry into consideration, the total number of displacements is 648. Meanwhile, the Lagrangian multiplier method is used to enforce the translational invariance constraint of $3^{\text {rd }}$ IFCs. $^{45}$ Here, a dense phonon q-grid of $15 \times 5 \times 5$ is used to calculate the lattice thermal conductivity of unfilled $\mathrm{Si}_{24}$ and fully filled $\mathrm{Si}_{24}$ with alkaline-earth metal atoms, and good convergence has been achieved.

\section{Results and discussion}

As shown in Fig. 1(a), the $\mathrm{Si}_{24}$ features regular array of nanosize pores (four pores with diameter about $0.5 \mathrm{~nm}$ in the primitive unit cell) as commonly seen in skutterudites and clathrates. Each of the pores is composed of eight-membered-rings of distorted $s p^{3}$ bonds, which is attributed to its two-step high-pressure precursor synthesis process. ${ }^{26}$ In the present calculation we mainly consider the structure fully filled with the group of alkaline-earth atoms. Fig. 1(b) shows the calculated lattice thermal conductivity (along $x x$ crystal direction) versus temperature for unfilled $\mathrm{Si}_{24}$, as well as fully filled $\mathrm{Si}_{24}$ with $\mathrm{Mg}\left(\mathrm{Mg} @ \mathrm{Si}_{24}\right)$, Ca $\left(\mathrm{Ca} @ \mathrm{Si}_{24}\right), \mathrm{Sr}\left(\mathrm{Sr} @ \mathrm{Si}_{24}\right)$, and $\mathrm{Ba}\left(\mathrm{Ba} @ \mathrm{Si}_{24}\right)$ atoms. Each system exhibits the well known $\kappa_{p} \sim 1 / T$ behavior, which is a typical character of intrinsic three phonon scattering resistance. It is not surprising that the lattice thermal conductivity is suppressed obviously when alkaline-earth atoms are inserted into the nanosize pores of $\mathrm{Si}_{24}$. At room temperature, the lattice thermal conductivity of pristine $\mathrm{Si}_{24}$ is reduced by approximately $87.3 \%$ for the sample filled with $\mathrm{Mg}$ atoms. Similar reduction of conductivity could also be found in the $\mathrm{Sr} @ \mathrm{Si}_{24}$ and $\mathrm{Ba} @ \mathrm{Si}_{24}$. The most striking results illustrated in Fig. 2(b) is that, comparing to $\mathrm{Mg}, \mathrm{Sr}$, and $\mathrm{Ba}$ atoms, inserting $\mathrm{Ca}$ atoms could cause more prominent suppression of lattice thermal conductivity of $\mathrm{Si}_{24}$ (the conductivity reduction is about $96.8 \%$ ) although they belong to the same group. Owing to the intrinsic geometric character, the lattice thermal conductivity of $\mathrm{Si}_{24}$ is anisotropic. From Table. 1 one can note that regardless of crystal direction, the insertion of alkaline-earth metal atoms, especially $\mathrm{Ca}$ atoms could dramatically reduce the thermal conductivity of $\mathrm{Si}_{24}$. For instance, the conductivity along the $z z$ crystal direction of $\mathrm{Ca} @ \mathrm{Si}_{24}$ is only about $0.59 \mathrm{~W} / \mathrm{mK}$, which is even smaller than that of amorphous silicon. These results suggest that among 

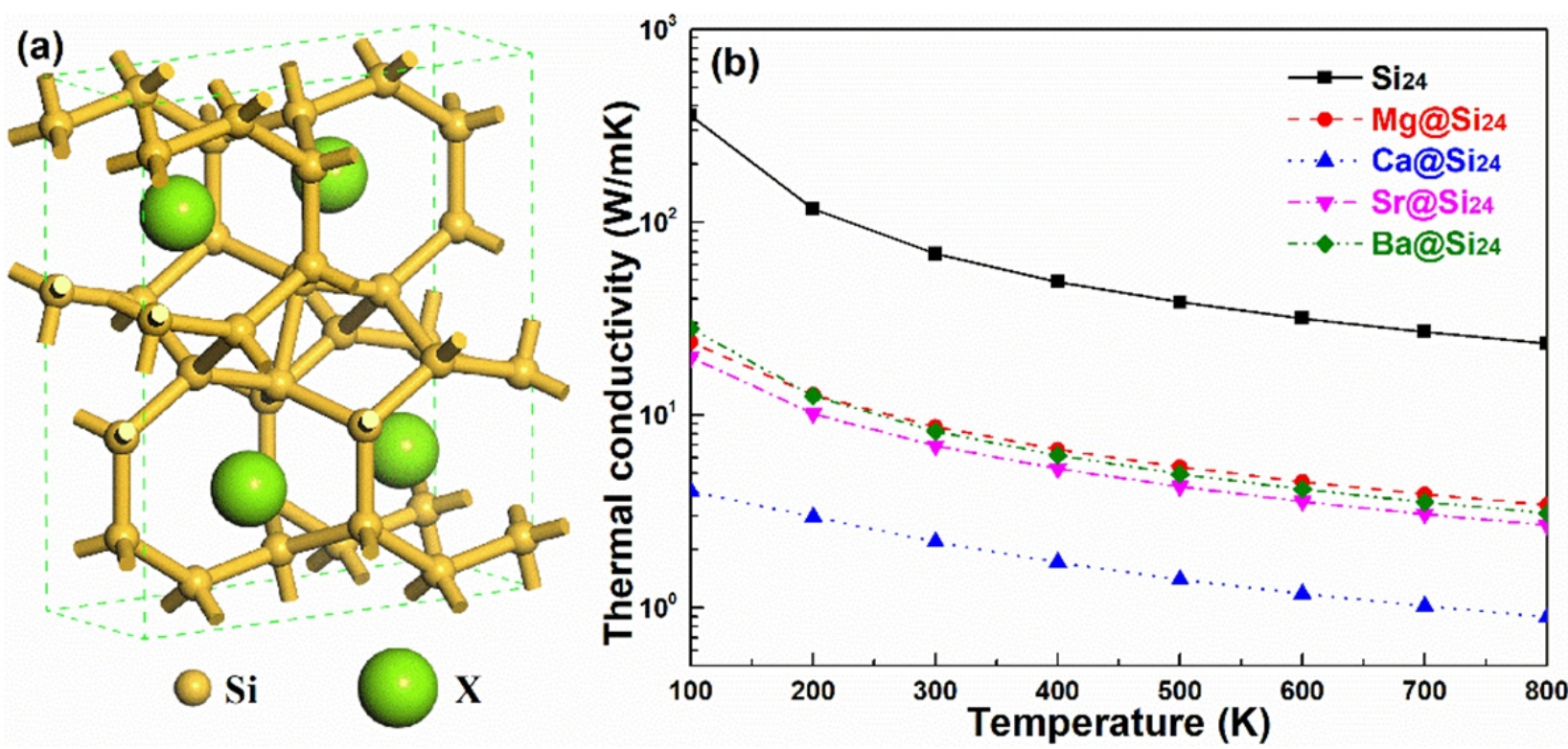

Fig. 1 (a) Schematic of atomic structure of fully filled open framework $\mathrm{Si}_{24}$ with alkaline-earth metal atoms $(\mathrm{X}=\mathrm{Mg}, \mathrm{Ca}, \mathrm{Sr}, \mathrm{Ba}),(\mathrm{b}) \mathrm{The}$ calculated temperature dependence of thermal conductivity of unfilled $\mathrm{Si}_{24}$ and fully filled $\mathrm{Si}_{24}$ with alkaline-earth metal atoms.
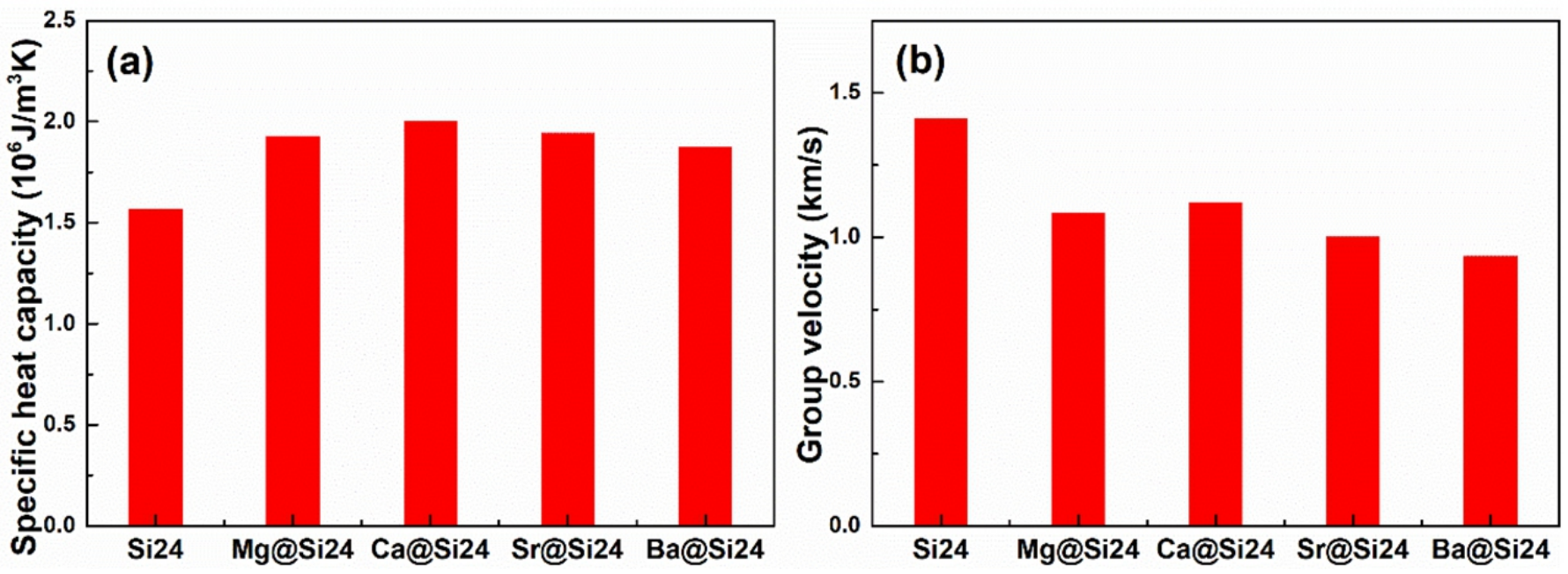

Fig. 2 (a) The specific heat capacity and (b) group velocity of unfilled $\mathrm{Si}_{24}$ and fully filled $\mathrm{Si}_{24}$ with alkaline-earth metal atoms.

alkaline-earth metal atoms, the $\mathrm{Ca}$ atom is the most suitable guest for decreasing the lattice thermal conductivity and boosting the corresponding thermoelectric performance of $\mathrm{Si}_{24}$.

To get insight into the underlying mechanism of such obvious suppression of thermal transport properties in fully filled $\mathrm{Si}_{24}$ with alkaline-earth metal atoms, we extract the mode-level contributions to the thermal conductivity including heat capacity $\left(C_{\lambda}\right)$, phonon group velocity $\left(v_{\lambda}\right)$, and phonon scattering rate $\left(1 / \tau_{\lambda}\right)$ of each phonon mode $\lambda$. Figs. 2(a) and 2(b) show the calculated heat capacity and average phonon group velocity of three acoustic phonon modes at room temperature. In contrast to the reduction of lattice thermal conductivity, the insertion of alkaline-earth metal atoms could slightly increase the heat capacity of $\mathrm{Si}_{24}$, which is attributed to the increase of phonon mode for the system filled with guest atoms. From Fig. 2(b) it can be found that the average phonon group velocities of $\mathrm{Mg} @ \mathrm{Si}_{24}, \mathrm{Ca} @ \mathrm{Si}_{24}, \mathrm{Sr} @ \mathrm{Si}_{24}$, and $\mathrm{Ba} @ \mathrm{Si}_{24}$ are approximately $22 \%, 20 \%, 29 \%$, and $33 \%$ lower than that of pristine $\mathrm{Si}_{24}$, respectively. This can be understood from the mode hybridization between the local vibrational of guest atoms and acoustic phonons of the host framework $\mathrm{Si}_{24}$, which is caused by the avoided crossing of the rattler modes. Such behavior will give rise to the flattened phonon branches (see more details in Fig. 5) as well as decrease of phonon group velocity. Nevertheless, the influences of both heat capacity and phonon group velocity are not enough to elucidate the evident reduction of lattice thermal conductivity observed in Fig. 1(b).

According to the definition of lattice thermal conductivity $\left(\kappa_{p}=\frac{1}{V} \Sigma\right.$, $C_{\lambda} v_{\lambda}^{2} \tau_{\lambda}$ ), until now the missing key factor not yet discussed is phonon anharmonic scattering rate (phonon relaxation time $\tau_{i}$ ) which might play a critical role on the reduction of thermal conductivity of $\mathrm{Si}_{24}$. Fig. 3 
Table 1 The calculated thermal conductivity $(300 \mathrm{~K})$ and volume of unfilled $\mathrm{Si}_{24}$ and fully filled $\mathrm{Si}_{24}$ with alkaline-earth metal atoms. The far right column is the quadratic coefficients of fitting line for the reduced atomic potential energy.

\begin{tabular}{cccccc}
\hline & $\boldsymbol{x} \boldsymbol{x}(\mathbf{W} / \mathbf{m K})$ & $\boldsymbol{y y}(\mathbf{W} / \mathbf{m K})$ & $z z(\mathbf{W} / \mathbf{m K})$ & Volume $\left(\boldsymbol{A}^{3}\right)$ & $\begin{array}{c}\text { Quadratic } \\
\text { coefficients }\end{array}$ \\
\hline $\mathbf{S i}_{\mathbf{2 4}}$ & 68.56 & 46.21 & 21.25 & 507.79 & \\
$\mathbf{M g} @ \mathbf{S i}_{24}$ & 8.70 & 4.45 & 4.01 & 511.83 & 9.72859 \\
$\mathbf{C a} @ \mathbf{S i}_{24}$ & 2.19 & 1.04 & 0.59 & 499.45 & 15.1285 \\
$\mathbf{S r} @ \mathbf{S i}_{24}$ & 6.92 & 3.72 & 3.77 & 516.94 & 10.20021 \\
$\mathbf{B a} @ \mathbf{S i}_{24}$ & 8.23 & 5.13 & 5.93 & 538.71 & 8.71171 \\
\hline
\end{tabular}

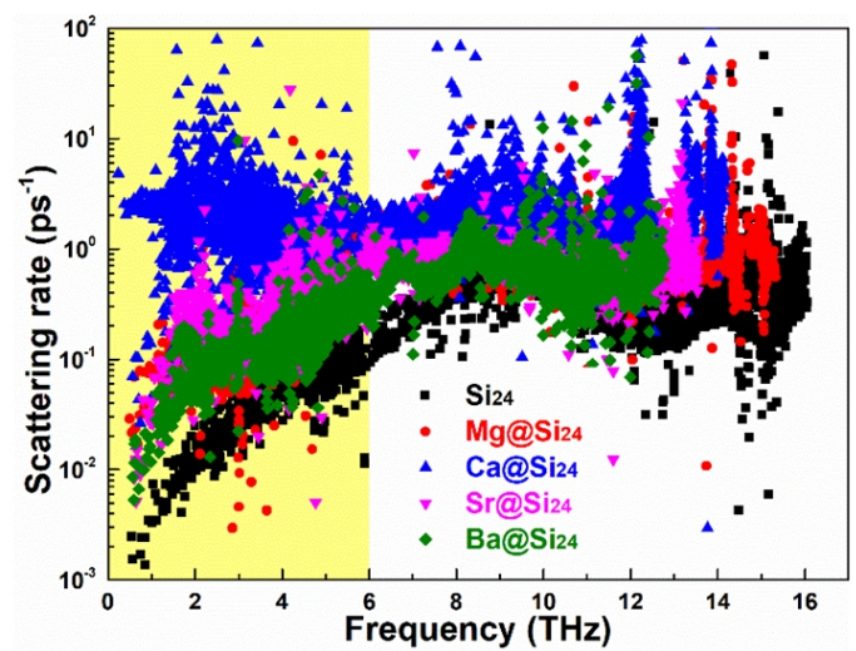

Fig. 3 The anharmonic scattering rates of unfilled $\mathrm{Si}_{24}$ and fully filled $\mathrm{Si}_{24}$ with alkaline-earth metal atoms. The significant increase in the phonon scattering rate of low frequency acoustic modes for the case of Ca filled $\mathrm{Si}_{24}$ is highlighted in yellow. shows the calculated three-phonon scattering rates $1 / \tau_{\lambda}$ at room temperature for unfilled $\mathrm{Si}_{24}$ and fully filled $\mathrm{Si}_{24}$ with alkaline-earth metal atoms. It can be seen that the phonon scattering rate of $\mathrm{Mg} @ \mathrm{Si}_{24}$ and $\mathrm{Ba} @ \mathrm{Si}_{24}$ is nearly one order of magnitude larger than that of pristine $\mathrm{Si}_{24}$ in the low and intermediate phonon frequency region (below $7 \mathrm{THz}$ ). The scattering rate of $\mathrm{Sr} @ \mathrm{Si}_{24}$ is little higher than those of $\mathrm{Mg} @ \mathrm{Si}_{24}$ or $\mathrm{Ba} @ \mathrm{Si}_{24}$. Clearly at most phonon frequency region, the scattering rate of $\mathrm{Ca} @ \mathrm{Si}_{24}$ is the highest among these systems, especially it is approximately three order of magnitude larger than that of pristine $\mathrm{Si}_{24}$ as frequency smaller than $4 \mathrm{THz}$. The higher phonon scattering rate correspond to smaller phonon relaxation time, and thus gives rise to less contributions of phonons to the lattice thermal conductivity. Therefore, the different behaviors observed in the phonon scattering rate are indeed another key factor for understanding the discrepancies in the thermal conductivity.

As we know that the phonon anharmonic scattering rate is determined by the three-phonon scattering strength and channel, to uncover the underlying mechanism, we firstly analyze the phonon scattering strength which is characterized by the Grüneisen parameters. From Fig. 4(a), it can be seen clearly that the mode weighted Grüneisen parameter of filled $\mathrm{Si}_{24}$ systems is obviously larger than that of pristine
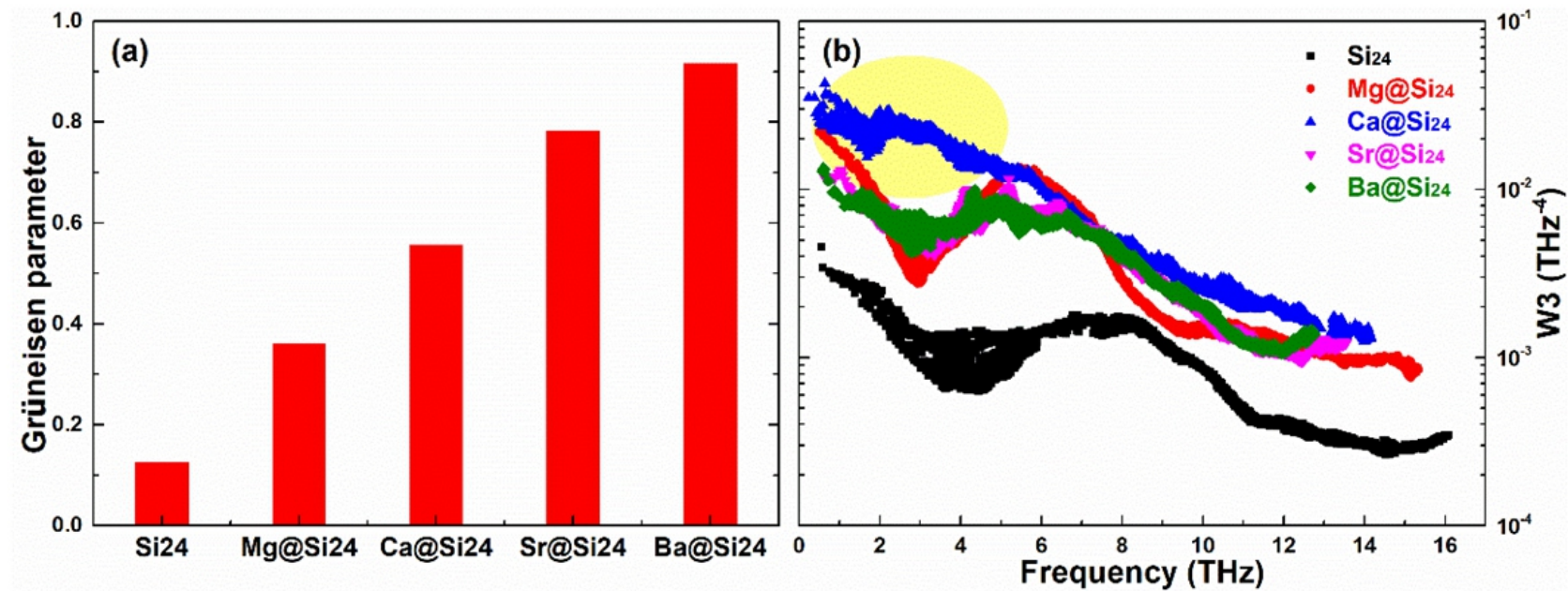

Fig. 4 (a) Mode cumulative Grüneisen parameter and (b) Phonon scattering phase space of unfilled $\mathrm{Si}_{24}$ and fully filled $\mathrm{Si}_{24}$ with alkaline-earth metal atoms. The significant increase in the phonon scattering phase space of low frequency acoustic modes for the case of Ca filled $\mathrm{Si}_{24}$ is highlighted in yellow. 

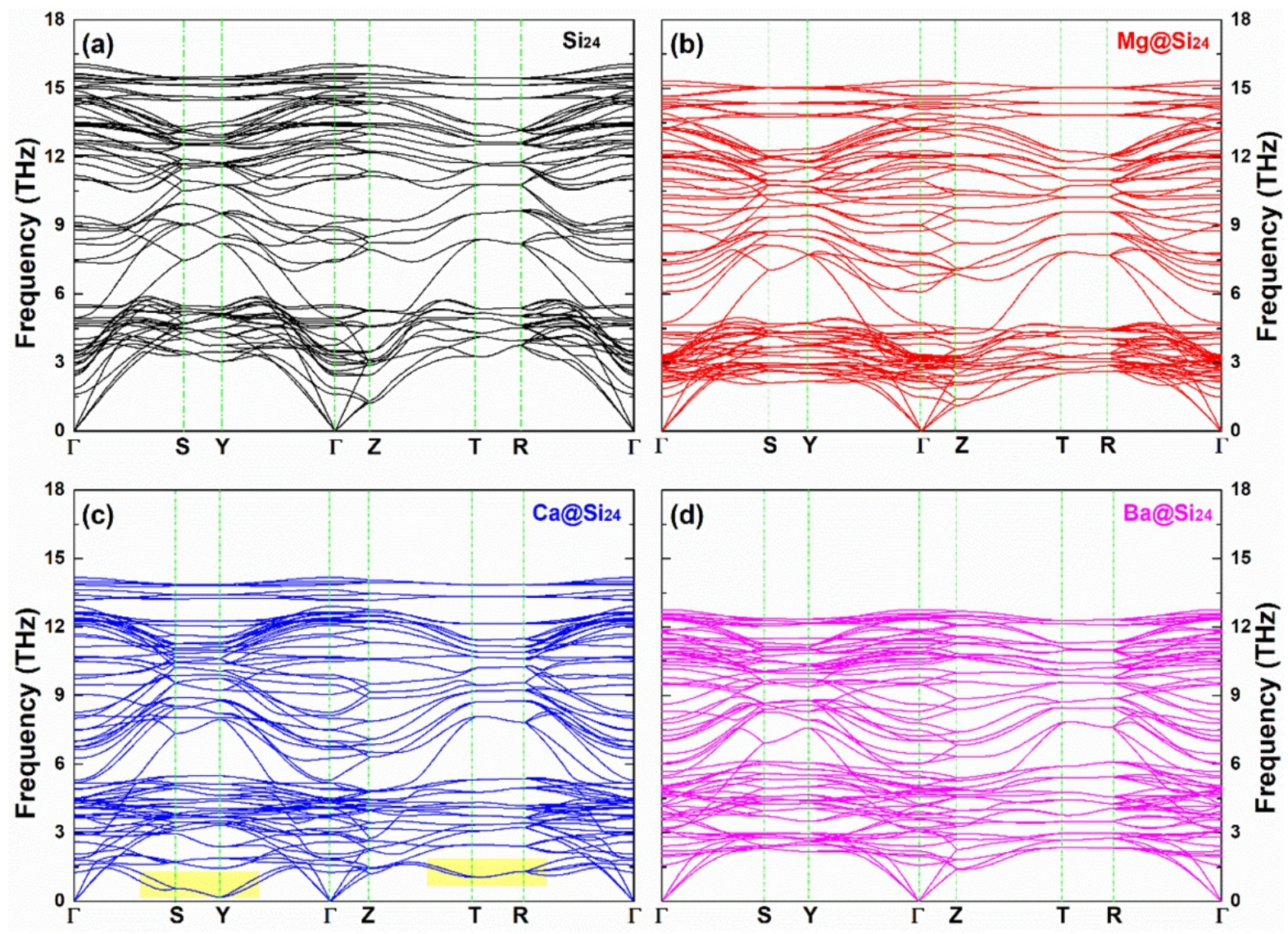

Fig. 5 Calculated phonon dispersion of (a) unfilled $\mathrm{Si}_{24}$ and fully filled $\mathrm{Si}_{24}$ with (b) $\mathrm{Mg}$, (c) $\mathrm{Ca}$, and (d) Ba. The low-lying optical phonon branches in the case of $\mathrm{Ca}$ filled $\mathrm{Si}_{24}$ are highlighted in yellow. The coordinates of high symmetry points are as follows: $\Gamma(0.00 .00 .0), \mathrm{S}(-0.50 .00 .0), \mathrm{Y}(-0.50 .50 .0)$,

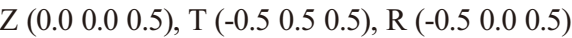

$\mathrm{Si}_{24}$, and it increases monotonously with the guest atoms mass. That is to say, inserting alkaline-earth metal atoms enhances the anharmonic scattering strength, and thus leads to the suppression of lattice thermal conductivity. However, such monotonous increase of Grüneisen parameter cannot explain the extremely low thermal conductivity of $\mathrm{Ca} @ \mathrm{Si}_{24}$, and similar reduction of conductivity of $\mathrm{Mg} @ \mathrm{Si}_{24}, \mathrm{Sr} @ \mathrm{Si}_{24}$, and $\mathrm{Ba} @ \mathrm{Si}_{24}$. Fig. 4(b) depicts the three-phonon scattering phase space which describes the available three-phonon scattering channels among all phonon modes. As can be seen, the scattering phase space of filled $\mathrm{Si}_{24}$ structures is distinctly several times larger than that of unfilled $\mathrm{Si}_{24}$ in the overall phonon frequency region. Among the four types of alkalineearth metal guest atoms, phonons in $\mathrm{Ca} @ \mathrm{Si}_{24}$ possess maximum scattering phase space, while that in $\mathrm{Ba} @ \mathrm{Si}_{24}$ and $\mathrm{Sr} @ \mathrm{Si}_{24}$ has less phase space especially in the low phonon frequency region (below 2.0 $\mathrm{THz}$ ). From the results presented above, one can obtain following conclusions. Firstly, the suppression of lattice thermal conductivity of filled $\mathrm{Si}_{24}$ systems is mainly attributed to the reduction of phonon group velocity and improvement of three-phonon anharmonic scattering (including both scattering strength and channels). This is quite different from the interpretation of previous studies which regards that only reduction of group velocity or enhancement of phonon scattering plays critical role on the decreasing of lattice thermal conductivity of guest atoms filled clathrates and skutterudites. Secondly, the analog reduction of thermal conductivity among $\mathrm{Mg} @ \mathrm{Si}_{24}, \mathrm{Sr} @ \mathrm{Si}_{24}$, and $\mathrm{Ba} @ \mathrm{Si}_{24}$ mainly stems from the competition between phonon scattering strength and channels. Finally, the key factor for the extremely low lattice thermal conductivity of $\mathrm{Ca} @ \mathrm{Si}_{24}$ is the large three-phonon scattering phase space.

Since the three-phonon scattering phase space is generally related with the phonon dispersion, in order to further understand the abnormal low lattice thermal conductivity of $\mathrm{Ca} @ \mathrm{Si}_{24}$, the corresponding phonon spectrum is plotted in Fig. 5. For comparison, the phonon dispersions of pristine $\mathrm{Si}_{24}, \mathrm{Mg} @ \mathrm{Si}_{24}$, and $\mathrm{Ba} @ \mathrm{Si}_{24}$ are also presented respectively. It can be seen clearly that the cutoff frequency of optical phonon modes (band width) is compressed upon loading the alkaline-earth metal atoms, and as the mass of guest atoms increases such compression becomes more obvious. Taking $\mathrm{Ba} @ \mathrm{Si}_{24}$ as an example, its band width is dramatically decreased to $12.78 \mathrm{THz}$ (about $82 \%$ of pristine $\mathrm{Si}_{24}$ $15.58 \mathrm{THz}$ ), indicating that the accessible heat conducting channels will be limited as $\mathrm{Ba}$ atoms are inserted in the void sites of the $\mathrm{Si}_{24}$ framework. Meanwhile, one can note that the phonon bands around 1.5 5.0 THz become flattened as guest atoms are filled in the cage of $\mathrm{Si}_{24}$. This is a common phenomenon in the phonon spectrum of guesthost systems and also the origin for reduction of phonon group velocity as shown in Fig. 2(b). In contrast to the $\mathrm{Mg} @ \mathrm{Si}_{24}$ and $\mathrm{Ba} @ \mathrm{Si}_{24}$, when the $\mathrm{Ca}$ atoms are introduced to the $\mathrm{Si}_{24}$, the phonon branches with frequency below $3.0 \mathrm{THz}$ become more dispersive, especially in the region around $\mathrm{S}$ to $\mathrm{Y}$ and $\mathrm{T}$ to $\mathrm{R}$ high symmetric points. In such dispersive phonon branches, the phonons will satisfy the requirement of energy and momentum conservation for scattering more easily. Accordingly, as shown in Fig. 4, the three phonon scattering phase space of $\mathrm{Ca} @ \mathrm{Si}_{24}$ is dramatically larger than that of $\mathrm{Si}_{24}$ filled with other alkaline-earth metal guest atoms.

To analyze behavior of phonon dispersion of fully filled $\mathrm{Si}_{24}$, we 
calculate the reduced atomic potential energy with respect to the displacement from equilibrium position, and the results are shown in Fig. 6. In the unit cell of each system, only one alkaline-earth metal guest atom is shifted from the equilibrium position (along $z z$ crystal direction), while keeping other atoms unchanged. As shown in Fig. 6, the atomic potential energy increases dramatically when the guest atom deviates from the corresponding equilibrium position. The amplification of potential energy shares analogous magnitude among $\mathrm{Mg} @ \mathrm{Si}_{24}$, $\mathrm{Sr} @ \mathrm{Si}_{24}$, and $\mathrm{Ba} @ \mathrm{Si}_{24}$, while that of $\mathrm{Ca} @ \mathrm{Si}_{24}$ is the maximal. Such discrepancy could also be demonstrated from the large quadratic coefficient (obtained by fitting the reduced atomic potential energy) of $\mathrm{Ca} @ \mathrm{Si}_{24}$ as indicated in Table I. The phonon dispersion is obtained through solving the eigenvalues of dynamical matrix constructed from the harmonic $2^{\text {nd }}$ IFCs which is second order partial derivative of potential energy. Therefore, the obvious increasing of atomic potential energy of $\mathrm{Ca} @ \mathrm{Si}_{24}$ with respect to the displacement of $\mathrm{Ca}$ atom could qualitatively explain the abnormal behavior of phonon dispersion shown in Fig. 5. Meanwhile, such obvious energy amplification also indicates that the atomic interaction in the $\mathrm{Ca} @ \mathrm{Si}_{24}$ is larger than that of $\mathrm{Si}_{24}$ filled with other alkaline-earth metal guest atoms.

Finally, in order to directly elucidate the interaction between the guest atoms and host framework, in Figs. 7(a) (c) we plot the

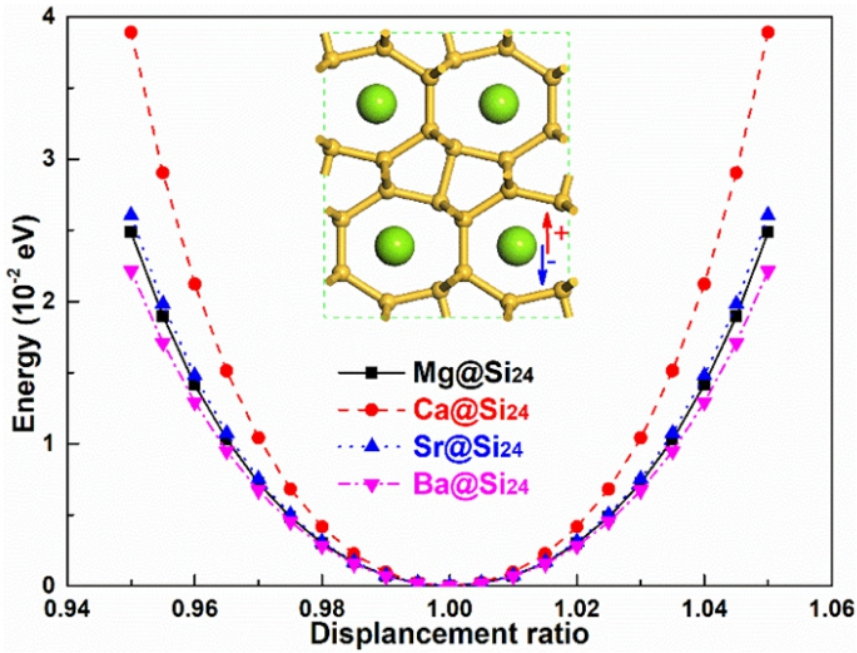

Fig. 6 The reduced potential energy of fully filled $\mathrm{Si}_{24}$ with respect to the displacement of guest atom from equilibrium position. Displacement ratio $\Delta Z$ is defined as: $\Delta Z=1.0+\left(Z-Z_{0}\right) / Z_{0}$, where $Z$ and $Z_{0}$ respectively represent the coordinate of shift position and equilibrium position.

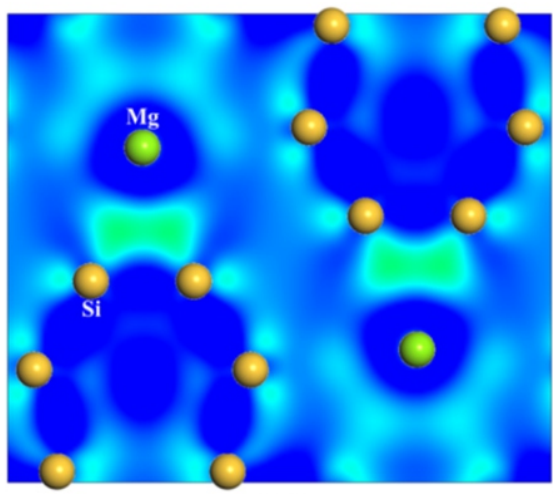

(a)

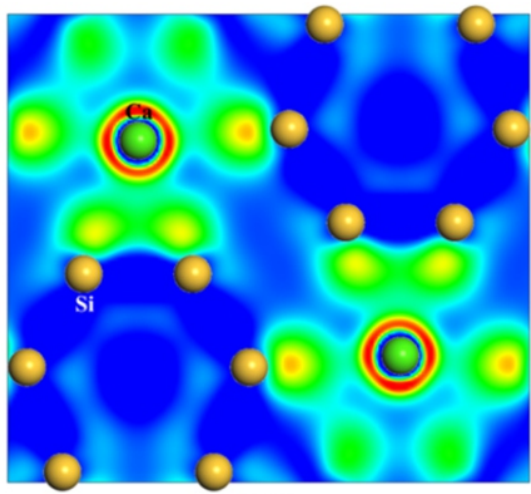

(b)

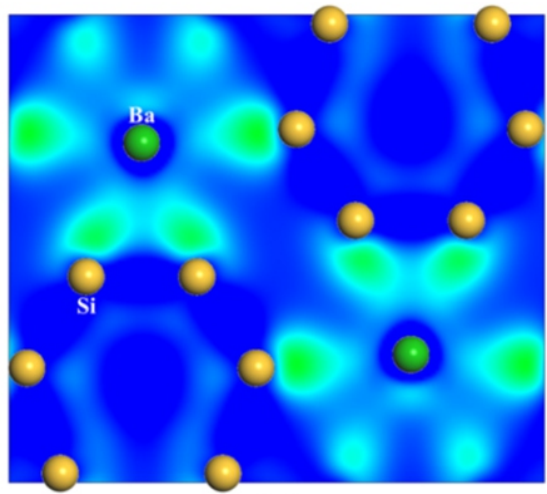

(c)

Fig. 7 Contour of deformation charge density within the (100) plane of fully filled $\mathrm{Si}_{24}$ with (a) $\mathrm{Mg}$, (b) Ca, and (c) Ba. The yellow and green balls are $\mathrm{Si}$ and guest atoms, respectively.

(a)

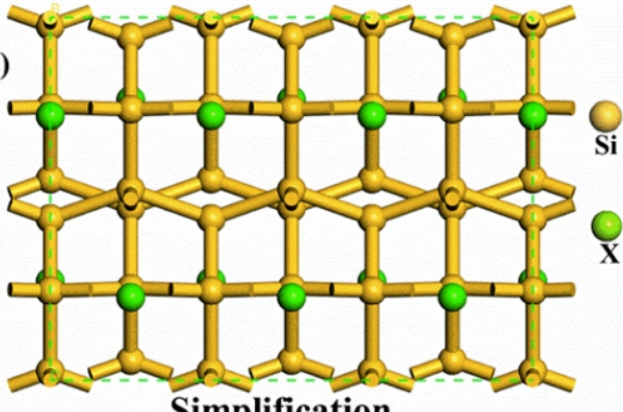

(b)

Simplification

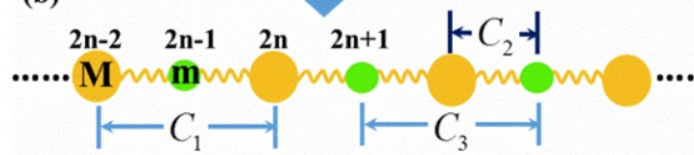

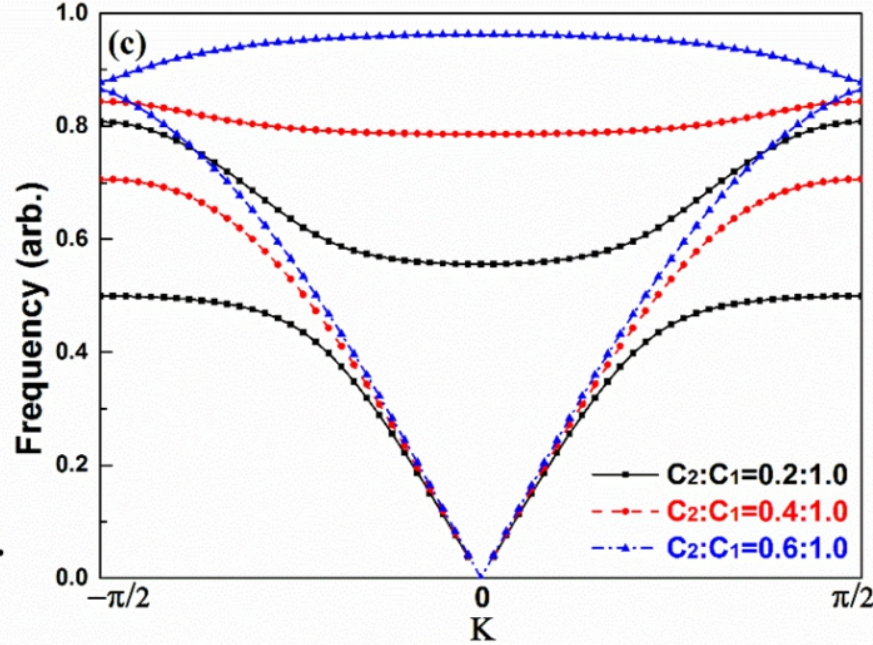

Fig. 8 (a) Side view of atomic structure of fully filled open framework $\mathrm{Si}_{24}$ with alkaline-earth metal atoms, (b) The corresponding simplified lattice chain model, (c) Calculated phonon dispersion of lattice chain model with different interatomic force constant ratio. 
Table 2 Comparison of average bader charge of $\mathrm{Si}$ and alkaline-earth metal atom in fully filled $\mathrm{Si}_{24}$ with $\mathrm{Mg}, \mathrm{Ca}, \mathrm{Sr}$, and $\mathrm{Ba}$.

\begin{tabular}{ccc}
\hline & $\mathbf{S i}(\mathbf{e})$ & $\mathbf{X}(\mathbf{e})$ \\
\hline $\mathbf{M g} @ \mathbf{S i}_{24}$ & {$[\mathrm{Ne}]+4.1844$} & {$[\mathrm{Ne}]+0.8935$} \\
$\mathbf{C a} @ \mathbf{S i}_{24}$ & {$[\mathrm{Ne}]+4.2171$} & {$[\mathrm{Ar}]+0.6972$} \\
$\mathbf{S r} @ \mathbf{S i}_{24}$ & {$[\mathrm{Ne}]+4.1891$} & {$[\mathrm{Kr}]+0.8652$} \\
$\mathbf{B a} @ \mathbf{S i}_{24}$ & {$[\mathrm{Ne}]+4.1839$} & {$[\mathrm{Xe}]+0.8968$} \\
\hline
\end{tabular}

deformation charge density (DCD) within the (100) plane of $\mathrm{Mg} @ \mathrm{Si}_{24}$, $\mathrm{Ca} @ \mathrm{Si}_{24}$, and $\mathrm{Ba} @ \mathrm{Si}_{24}$, respectively. From the distribution of DCD, it is clearly seen that as the alkaline-earth metal atoms are filled in the cage, part of charge of guest atoms will transfer to the surrounding $\mathrm{Si}$ atoms of the host framework. Among the $\mathrm{Mg}, \mathrm{Ca}$, and $\mathrm{Ba}$ atoms, the charge transformation in the $\mathrm{Ca} @ \mathrm{Si}_{24}$ structure is the most conspicuous. This phenomenon can be also demonstrated from the Bader charge analysis shown in Table II. One can note that the loss of charge of Ca atoms is about 0.3 e/atom, while that of other alkaline-earth metal atoms is merely $0.1 \mathrm{e} /$ atom. Such evident charge transformation suggests that the interaction strength between the $\mathrm{Ca}$ and $\mathrm{Si}$ atoms is far larger than that between the $\mathrm{Mg} / \mathrm{Sr} / \mathrm{Ba}$ and $\mathrm{Si}$ atoms. Meanwhile, such strong interaction makes the distortion of $\mathrm{Si}$ framework become more severe (the volume abnormally shrinks as $\mathrm{Ca}$ atoms are filled in the cage of $\mathrm{Si}_{24}$, and the result is shown in Table I). Generally, it is well known that the stronger interaction strength among atoms is, the more dispersive phonon branches exist. Consequently, the $\mathrm{Si}_{24}$ framework filled with $\mathrm{Ca}$ atoms possesses more dispersive phonon dispersion and large scattering phase space, and thus leads to the low lattice thermal conductivity compared with the system containing other alkaline-earth metal guest atoms.

Before closing, it is worth to point out that, this abnormal thermal phenomenon could also be illustrated by a simple lattice chain model shown in Figs. 8(a) and (b). In this model, the yellow lattices represent $\mathrm{Si}_{24}$ frameworks and their interaction is described by force constant $C_{1}$, while the green lattices represent the alkaline-earth metal fillers and the interactions between guest and host lattices and guest and guest lattices are respectively described by force constant $C_{2}$ and $C_{3}$. Based on this simple chain model, the equation of motion is expressed as:

$$
\begin{aligned}
& M \frac{\partial^{2} u_{2 n}}{\partial x^{2}}=-C_{2}\left(2 u_{2 n}-u_{2 n+1}-u_{2 n-1}\right)-C_{1}\left(2 u_{2 n}-u_{2 n+2}-u_{2 n-2}\right) \\
& m \frac{\partial^{2} u_{2 n+}}{\partial x^{2}}=-C_{2}\left(2 u_{2 n+1}-u_{2 n+2}-u_{2 n}\right)-C_{3}\left(2 u_{2 n+1}-u_{2 n+3}-u_{2 n-1}\right)
\end{aligned}
$$

here $u$ denotes the corresponding displacement. This equation set has following solution in the form of a traveling wave with different amplitudes A, B on alternate lattices,

$$
\begin{aligned}
& u_{2 n}=A e^{i[\omega t-2 n a K]} \\
& u_{2 n+1}=B e^{i[\omega t-(2 n+1) a K]}
\end{aligned}
$$

Substituting Eq. (2) in Eq. (1) we have

$$
\begin{aligned}
& -\omega^{2} M A=-C_{2}\left(2 A-B e^{i a K}-B e^{-i a K}\right)-C_{1}\left(2 A-A e^{i 2 a K}-A e^{-i 2 a K}\right) \\
& -\omega^{2} m B=-C_{2}\left(2 B-A e^{i a K}-A e^{-i a K}\right)-C_{3}\left(2 B-B e^{i 2 a K}-B e^{-i 2 a K}\right)
\end{aligned}
$$

Considering $e^{i a K}+e^{-i a K}=2 \cos (a K)$, the Eq. (3) could be written as:

$$
\begin{aligned}
& -\omega^{2} M A=-2 C_{2} A+2 C_{2} B \cos (a K)-2 C_{1} A+2 C_{1} A \cos (2 a K) \\
& -\omega^{2} m B=-2 C_{2} B+2 C_{2} A \cos (a K)-2 C_{3} B+2 C_{3} B \cos (2 a K)
\end{aligned}
$$

The homogeneous linear equations have a solution only if the determinant of the coefficients of the unknowns A, B vanishes:

$$
\left|\begin{array}{cc}
\omega^{2} M-2 C_{2}-2 C_{1}++2 C_{1} \cos (2 a K) & 2 C_{2} \cos (a K) \\
2 C_{2} \cos (a K) & \omega^{2} m-2 C_{2}-2 C_{3}+2 C_{3} \cos (2 a K)
\end{array}\right|=0
$$

This equation could be simply expressed as:

$$
\left|\begin{array}{cc}
\omega^{2}+M_{A 1} & M_{B 1} \\
M_{A 2} & \omega^{2}+M_{B 2}
\end{array}\right|=0
$$

or $\omega^{4}+\left(M_{A 1}+M_{B 2}\right) \omega^{2}+M_{A 1} M_{B 2}-M_{B 1} M_{A 2}=0$, where

$M_{A 1}=\left[-2 C_{2}-2 C_{1}++2 C_{1} \cos (2 a K)\right] / M, M_{B 1}=2 C_{2} \cos (a K) / M$,

$M_{A 2}=2 C_{2} \cos (a K) / m$ and $M_{B 2}=\left[-2 C_{2}-2 C_{3}+2 C_{3} \cos (2 a K)\right] / m$.

Through solving this equation, we can get the phonon dispersion of the simplified lattice chain model:

$$
\omega_{ \pm}^{2}=\frac{-\left(M_{A 1}+M_{B 2}\right) \pm \sqrt{\left(M_{A 1}+M_{B 2}\right)^{2}-4\left(M_{A 1} M_{B 2}-M_{B 1} M_{A 2}\right)}}{2}
$$

Here the interaction between the guest and guest lattices $C_{3}$ is assumed to be zero for simplification (this is reasonable since in real materials the guest atoms are separated far away), and the value of $M$ and $m$ is respectively set as the mass of $\mathrm{Si}_{24}$ framework and filling $\mathrm{Ca}$ atoms. From Fig. 8(c), one can clearly note that when the interaction strength between the guest and host lattice increases, the optical phonon branch around the edge of Brillouin zone gradually shift to low frequency region and the corresponding gap between acoustic and optical phonon branches decreases simultaneously. This behavior will make the requirement of energy and momentum conservation for phonon scattering more likely, and thus enhancing the scattering phase space and leading to low thermal conductivity. Such simple lattice chain model qualitatively reproduces the thermal transport in the $\mathrm{Ca} @ \mathrm{Si}_{24}$ and further demonstrates that the strong harmonic atomic interaction between $\mathrm{Ca}$ and $\mathrm{Si}$ atoms is the origin for the abnormal low lattice thermal conductivity. 


\section{Conclusions}

To summarize, taking open framework $\mathrm{Si}_{24}$ as an example, we systematically investigate the lattice thermal conductivity of this new structure filled with alkaline-earth guest atoms through combining firstprinciples calculation and phonon Boltzmann transport theory. The calculations show that the lattice thermal conductivity is obviously decreased as guest atoms are inserted in the void sites of the $\mathrm{Si}_{24}$ framework, which is a common phenomenon occurring in guest-host systems. However, it is interesting to find that the thermal conductivity of this new filler structure presents a prominent element dependency. The $\mathrm{Ca}$ guest atoms exhibit an unique character that the thermal conductivity of Ca filled $\mathrm{Si}_{24}$ framework is generally 3 to 10 times lower than that with other alkaline-earth atom fillers, and the value along $z z$ crystal direction $(0.59 \mathrm{~W} / \mathrm{mK})$ is even lower than that of amorphous silicon. By comparing the key contributors of the lattice thermal conductivity at the phonon mode level, we find that distinct difference of lattice thermal conductivity between $\mathrm{Ca} @ \mathrm{Si}_{24}$ and $\mathrm{Ma}(\mathrm{Sr}, \mathrm{Ba}) @ \mathrm{Si}_{24}$ mainly originates from the three-phonon scattering phase space. Further analysis reveals that the strong harmonic atomic interaction among guest and host atoms in $\mathrm{Ca} @ \mathrm{Si}_{24}$ is the key and governing parameter for the dramatically suppressed thermal conductivity, which could lead to dispersive phonon branches in low frequency region and enhancement of three-phonon scattering channels. The analysis of deformation charge density distribution further establishes the correlation between the suppressed thermal transport phenomenon and the nature of interatomic interactions. Finally, through simplifying the guest-host system into a lattice chain model, we further demonstrate that the large suppression of lattice thermal conductivity in $\mathrm{Ca} @ \mathrm{Si}_{24}$ is mainly attributed to the strong interaction between guest $(\mathrm{Ca})$ and host $(\mathrm{Si})$ atoms. These findings provide insight into the new physical mechanism for the more severely reduced thermal conductivity of $\mathrm{Ca} @ \mathrm{Si}_{24}$ as compared with other alkaline-earth guest atoms and pave the way for improving the thermoelectric efficiency of $\mathrm{Si}_{24}$ by concurrently reducing lattice thermal conductivity and enhancing electrical properties.

\section{Acknowledgement}

This work is supported by the National Natural Science Foundation of China (No. 11304262), Scientific Research Fund of Hunan Provincial Education Department (No. 17B252), Natural Science Foundation of Hunan Province (Grant No. 2016JJ3118), and Program for Changjiang Scholars and Innovative Research Team in University (IRT13093). M.H. acknowledges the start-up fund from the University of South Carolina.

\section{References}

1. L. El Chaar, L. A. lamont and N. El Zein, Renew. Sust. Energ. Rev., 2011, 15, 2165-2175.

2. B. Parida, S. Iniyan and R. Goic, Renew. Sust. Energ. Rev., 2011, 15, 16251636.

3. L. E. Bell, Science, 2008, 321, 1457.

4. G. J. Snyder and E. S. Toberer, Nat. Mater, 2008, 7, 105.

5. J. Yang, L. Xi, W. Qiu, L. Wu, X. Shi, L. Chen, J. Yang, W. Zhang, C. Uher and D. J. Singh, NPJ Comput. Mater, 2016, 2, 15015.

6. X. Y. Mi, X. Yu, K. L. Yao, X. Huang, N. Yang and J. T. Lü, Nano Lett., 2015, 15, 5229-5234

7. L. Yang, N. Yang and B. Li, Int. J. Heat Mass Tran., 2016, 99, 102-106.

8. G. Xie, D. Ding and G. Zhang, Advances in Physics: X, 2018, 3, 1480417.

9. T. M. Tritt, Аnпu. Rev. Mater. Res., 2011, 41, 433-448.
10. A. I. Hochbaum, R. Chen, R. D. Delgado, W. Liang, E. C. Garnett, M. Najarian, A. Majumdar and P. Yang, Nature, 2008, 451, 163.

11. A. I. Boukai, Y. Bunimovich, J. Tahir-Kheli, J. K. Yu, W. A. Goddard Iii and J. R. Heath, Nature, 2008, 451, 168.

12. S. K. Bux, R. G. Blair, P. K. Gogna, H. Lee, G. Chen, M. S. Dresselhaus, R. B. Kaner and J. P. Fleurial, Adv. Funct. Mater., 2009, 19, 2445-2452.

13. D. A. Broido, M. Malorny, G. Birner, N. Mingo and D. A. Stewart, Appl. Phys. Lett., 2007, 91, 231922.

14. H. J. Xiang, B. Huang, E. Kan, S. H. Wei and X. G. Gong, Phys. Rev. Lett., 2013, 110, 118702.

15. S. Botti, J. A. Flores-Livas, M. Amsler, S. Goedecker and M. A. L. Marques, Phys. Rev. B, 2012, 86, 121204.

16. Q. Wang, B. Xu, J. Sun, H. Liu, Z. Zhao, D. Yu, C. Fan and J. He, J. Am Chem. Soc., 2014, 136, 9826-9829.

17. C. He, C. Zhang, J. Li, X. Peng, L. Meng, C. Tang and J. Zhong, Phys. Chem. Chem. Phys., 2016, 18, 9682-9686.

18. I. H. Lee, J. Lee, Y. J. Oh, S. Kim and K. J. Chang, Phys. Rev. B, 2014, 90, 115209

19. J. Crain, G. J. Ackland, J. R. Maclean, R. O. Piltz, P. D. Hatton and G. S Pawley, Phys. Rev. B, 1994, 50, 13043-13046.

20. R. H. Wentorf and J. S. Kasper, Science, 1963, 139, 338-339.

21. F. Yoshitaka, K. Takashi, S. Susumu, M. Takashi and O. Atsushi, New J. Phys., 2008, 10, 083001.

22. J. S. Kasper, P. Hagenmuller, M. Pouchard and C. Cros, Science, 1965, 150 1713-1714.

23. G. Xie, Z. Ju, K. Zhou, X. Wei, Z. Guo, Y. Cai and G. Zhang, NPJ Comput Mater, 2018, 4, 21.

24. D. Ma, H. Ding, H. Meng, L. Feng, Y. Wu, J. Shiomi and N. Yang, Phys. Rev. B, 2016, 94, 165434.

25. T. Takabatake, K. Suekuni, T. Nakayama and E. Kaneshita, Rev. Mod. Phys. 2014, 86, 669-716

26. D. Y. Kim, S. Stefanoski, O. O. Kurakevych and T. A. Strobel, Nat. Mater, 2014, 14, 169.

27. K. Chae, S. H. Kang, S. M. Choi, D. Y. Kim and Y. W. Son, Nano Letters, 2018, 18, 4748-4754.

28. T. Ouyang, P. Zhang, H. Xiao, C. Tang, J. Li, C. He and J. Zhong, J. Phys. D: Appl. Phys., 2017, 50, 425501.

29. M. Christensen, A. B. Abrahamsen, N. B. Christensen, F. Juranyi, N. H Andersen, K. Lefmann, J. Andreasson, C. R. H. Bahl and B. B. Iversen, Nat. Mater, 2008, 7, 811 .

30. S. Pailhès, H. Euchner, V. M. Giordano, R. Debord, A. Assy, S. Gomès, A. Bosak, D. Machon, S. Paschen and M. de Boissieu, Phys. Rev. Lett., 2014, 113, 025506

31. M. Zebarjadi, K. Esfarjani, J. Yang, Z. F. Ren and G. Chen, Phys. Rev. B, 2010, 82, 195207.

32. B. Huang and M. Kaviany, Acta Mater., 2010, 58, 4516-4526.

33. M. M. Koza, M. R. Johnson, R. Viennois, H. Mutka, L. Girard and D. Ravot, Nat. Mater, 2008, 7, 805.

34. T. Tadano, Y. Gohda and S. Tsuneyuki, Phys. Rev. Lett., 2015, 114, 095501.

35. J. Y. Yang, L. Cheng and M. Hu, Appl. Phys. Lett., 2017, 111, 242101.

36. P. Norouzzadeh, C. W. Myles and D. Vashaee, Phys. Rev. B, 2017, 95, 195206.

37. Y. Wang, H. Yang, W. Qiu, J. Yang, J. Yang and W. Zhang, Phys. Rev. B, 2018, 98, 054304.

38. T. Tadano and S. Tsuneyuki, Phys. Rev. Lett., 2018, 120, 105901.

39. G. Kresse and J. Furthmüller, Phys. Rev. B, 1996, 54, 11169-11186.

40. P. E. Blöchl, Phys. Rev. B, 1994, 50, 17953-17979.

41. G. Kresse and D. Joubert, Phys. Rev. B, 1999, 59, 1758-1775.

42. D. M. Ceperley and B. J. Alder, Phys. Rev. Lett., 1980, 45, 566-569.

43. W. Li, J. Carrete, N. A. Katcho and N. Mingo, Comput. Phys. Commun., 2014, 185, 1747-1758.

44. A. Togo and I. Tanaka, Scripta Mater, 2015, 108, 1-5.

45. W. Li, L. Lindsay, D. A. Broido, D. A. Stewart and N. Mingo, Phys. Rev. B, 2012, 86, 174307. 